\title{
Synthesis of Core-Shell Type Gel Beads Consisting of Two Different Poly $(N$-alkylacrylamide) Gel Layers, and Their Thermosensitive Behavior
}

\author{
By Takashi IIZAWA, * Akihiro TERAO, Tomomi ABE, Machiko OHUChIDA, and Yoshinobu MatsuURA
}

Mm-size monodispersed poly(acrylic acid) gel-1,8-diazabicyclo[5,4,0]undec-7-ene (DBU) salt (DAA) beads were prepared via a two-step procedure involving the sedimentation copolymerization of acrylic acid with 0.5 mol $\%$ of MBAA and neutralization of the resulting gel with excess DBU in methanol. When the resulting DAA beads were placed in $N$-methyl-2pyrrolidone containing an excess of alkylamine and triphenylphosphine, selective amidation occurred from the outside to give the corresponding DAA-poly( $N$-alkylacrylamide) (PNAA) core-shell type gel bead, consisting of an unreacted DAA core and an amidated shell layer. The thickness of the shell layer could be controlled by the reaction time. Second amidation of the obtained DAA-PNAA core-shell type gel bead with another alkylamine resulted in a novel mm-size monodispersed core-shell type bead consisting of two different PNAA layers, poly( $N$ - $n$-propylacrylamide) and poly $(N$-isopropylacrylamide). The swelling and de-swelling of the gel bead shell layer and core occurred independently in response to temperature changes. The amidation of the DAA beads and the swelling/de-swelling of the amidated gel beads occurred isotropically, and they maintained their spherical shapes.

KEY WORDS: Double Thermosensitive Gel / Sedimentation Polymerization / Poly(Nalkylacrylamide) Gel / Core-Shell Type Gel /

Sedimentation polymerization has attracted attention as a synthetic method for the production of mm-size monodispersed beads. ${ }^{1}$ These beads can usually be obtained from a classification of the beads prepared by suspension polymerization. The yield is so low that pellet-type gels have often been used instead of these beads. However, sedimentation polymerization can effectively produce mm-size monodispersed beads. The polymerization mechanism has been described as follows: when an aqueous monomer solution is injected dropwise to an immiscible heat medium (liquid paraffin or silicone oil), surface gelation of droplets descending in the medium takes place to give a stable skin, which prevents coalescence at the base of the reactor, and the polymerization is completed at the base.

Improved sedimentation polymerization systems such as oilin-water-in-oil sedimentation polymerization; ${ }^{2}$ compressed fluid sedimentation polymerization using supercritical carbon dioxide as a heat medium; ${ }^{3}$ nonaqueous sedimentation polymerization using an aprotic polar solvent; ${ }^{4}$ application to the crosslinking reaction of poly(vinyl alcohol) with glutaraldehyde $;^{5}$ and, polycondensation of tetraethyl orthosilicate ${ }^{6}$ have been investigated. Mm-size functional polymer beads such as composite polymer beads, ${ }^{5}$ interpenetrating polymer network beads, ${ }^{5}$ and porous polymer beads ${ }^{2,7}$ have been prepared by these methods. However, the chemical modification of beads prepared by sedimentation polymerization has rarely been reported. In the sedimentation polymerization of an aqueous $N$ alkylacrylamide solution, phase-separation occurred to give porous hollow particles. ${ }^{7,8}$ These results support the polymerization mechanism described above, and suggest that polymer- ization could afford a bead with a non-uniform internal structure. Thus, the question arises as to whether beads prepared by sedimentation polymerization could be available as starting materials for chemical modification.

A novel synthetic method was recently proposed for the production of mm-size core-shell gels by the heterogeneous reaction of polymer gels. ${ }^{9}$ This was the reaction of cylindrical poly(acrylic acid) gel-1,8-diazabicyclo[5,4,0]undec-7-ene (DBU) salt (DAA) with alkylamine using triphenylphosphine (TPP) as an activating reagent (Scheme 1). When the starting gel does not swell in a reaction solvent and the highly reacted gel becomes swelled in the solvent, and the chemical reaction is relatively fast, the reaction take place on the surface of the unreacted core to give a core-shell type gel containing an unreacted core and a highly reacted (swelled) shell layer. The unreacted core shrinks with increasing reaction time. The shrinking rate of the unreacted core is markedly affected by the

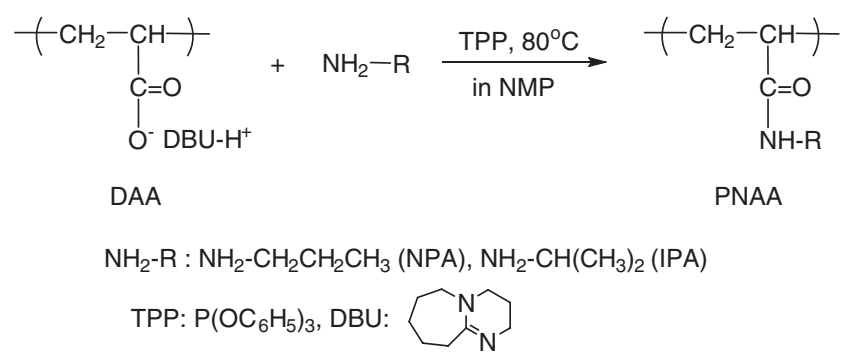

Scheme 1. Synthesis of a PNAA gel via the reaction of DAA with NPA and IPA.

Department of Chemical Engineering, Graduate School of Engineering, Hiroshima University, Kagamiyama, Higashi-Hiroshima 739-8527, Japan *To whom correspondence should be addressed (Tel: +81-82-424-7711, Fax: +81-82-424-5494, E-mail: tiizawa@ hiroshima-u.ac.jp). 


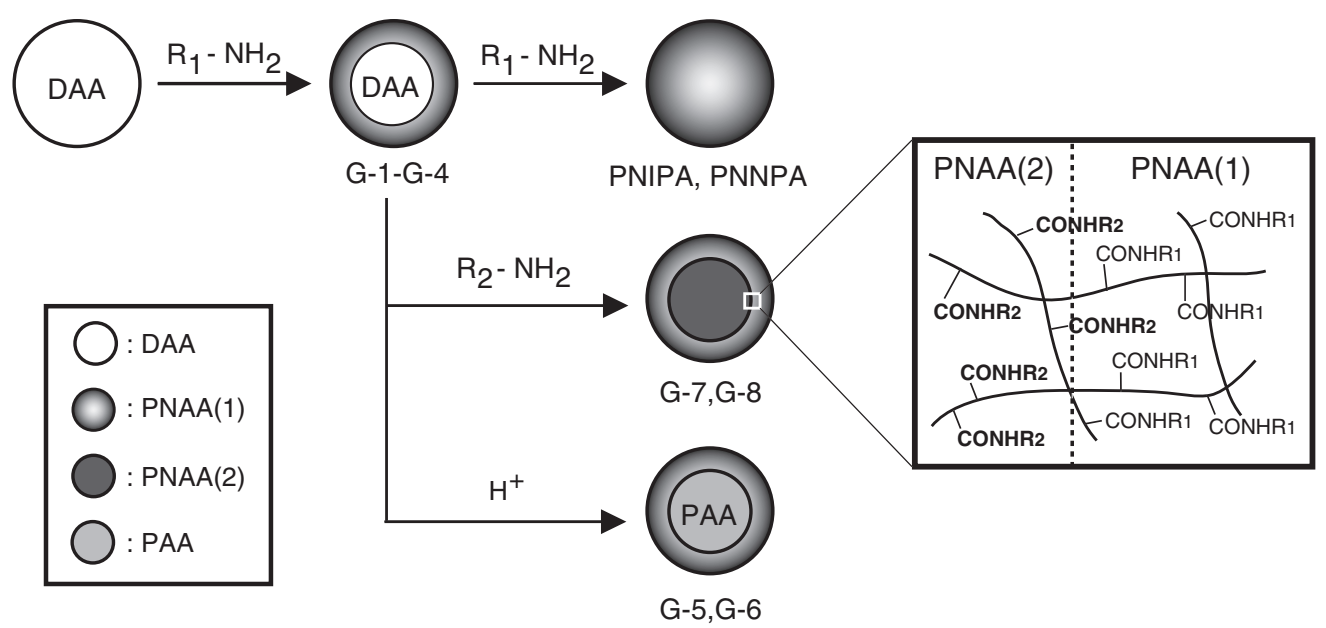

Scheme 2. Synthesis of a PNAA gel bead, DAA-PNAA core-shell type gel bead (G-1-G-4), PAA-PNAA core-shell type gel bead (G-5, G-6), and PNAA(2)PNAA(1) core-shell type gel bead (G-7, G-8).

mass transfer rate of reactants and the solvent through the reacted shell layer. This reaction mechanism has been characterized as similar to the shrinking-core model. ${ }^{10}$

Further amidation of the unreacted core with a different alkylamine afforded a PNAA(2)-PNAA(1) core-shell type gel consisting of two different thermosensitive poly $(N$-alkylacrylamide): PNAA(2) and PNAA(1) layers. ${ }^{11}$ Since the resulting core-shell type gel has essentially the same main chain structure and shape as the original DAA sample, it has a defined structure - a continuous network and a boundary between two layers. This core-shell type gel corresponds to well-controlled crosslinking block copolymers with double thermosensitivity. Double thermosensitive block copolymers such as $\operatorname{poly}(N$-isopropylacrylamide-block-sulfobetaine $),{ }^{12}$ poly[2-( $N$-morpholino)ethyl methacrylate-block-sulfobetaine $],{ }^{13}$ and poly $(N, N \text {-diethylacrylamide-block-sulfobetaine })^{14}$ showed independent thermosensitive behavior of each block, which caused dramatically changing associations between micelle and reverse micelle with temperature changes. This core-shell type gel also showed interesting swelling/deswelling behavior. For example, the resulting asymmetric core-shell gels showed a marked deformation between bending and stretching in response to water temperature changes. ${ }^{11,15}$ Unfortunately, the reaction and the swelling/de-swelling behavior of the cylindrical gels cannot be precisely observed, because the shapes become distorted during the reaction and the swelling/de-swelling.

In a previous paper, ${ }^{16}$ the authors reported the heterogeneous esterification of poly(2-hydroxyethyl acrylate) (PHEA) gel beads resulting from sedimentation polymerization with acetic anhydride to give fine spherical core-shell type beads containing an esterified shell and an unreacted core. This study reports the synthesis of DAA beads from the sedimentation polymerization of acrylic acid and the heterogeneous amidation of the resulting beads with alkylamine (Scheme 2). The reaction proceeded through fine spherical core-shell type gels, and the resulting gels maintained their spherical shape during the swelling/de-swelling if the starting bead was uniform and the reaction maintained a mechanism similar to the shrinking-core model. $^{10}$

\section{EXPERIMENTAL}

\section{Materials}

Acrylic acid (AA) and solvents were distilled prior to use. Commercial alkylamines such as $n$-propylamine (NPA) and isopropylamine (IPA) were used as purchased. $N, N^{\prime}$-methylenebisacrylamide (MBAA), DBU, and TPP were used without further purification.

\section{Apparatus}

IR spectra were obtained using a Perkin Elmer model IR700 spectrophotometer.

\section{Synthesis of DAA Beads}

DAA beads were prepared via a two-step procedure involving the sedimentation copolymerization of AA with $0.5 \mathrm{~mol} \%$ of MBAA and neutralization of the resulting gel with excess DBU in methanol, as follows. AA $(5.0 \mathrm{~g}, 694 \mathrm{mmol})$, MBAA $(53.5 \mathrm{mg}, 0.347 \mathrm{mmol})$, and potassium persulfate $(93.9 \mathrm{mg}, 0.347 \mathrm{mmol})$ were dissolved in water $(15.0 \mathrm{~g})$ at $20{ }^{\circ} \mathrm{C}$ under nitrogen atmosphere. Silicone oil (GE Toshiba Silicones Co., kinematic viscosity: $3000 \mathrm{~mm}^{2} \mathrm{~s}^{-1}$ at $25^{\circ} \mathrm{C}$ ) was charged into a washing bottle in a water bath at $70^{\circ} \mathrm{C}$ and nitrogen gas was bubbled into the silicone oil over $3 \mathrm{~h}$. The monomer solution was injected dropwise into the silicone oil using a syringe (external diameter $1.5 \mathrm{~mm}, 90^{\circ}$ tip). It was allowed to stand for $12 \mathrm{~h}$ at $80^{\circ} \mathrm{C}$ under a nitrogen stream. The resulting beads were immersed into a large amount of methanol containing excess DBU, then let stand for $24 \mathrm{~h}$. The swelled beads were washed with methanol by a Soxhlet extractor to wash away unreacted substances. The beads were dried carefully and slowly, and finally dried in vacuo at $60^{\circ} \mathrm{C}$ to a constant weight. The average diameters $\left(\overline{2 R_{0}}\right)$ and the relative 
standard deviation $(\delta)$ of the obtained beads were $2.18 \mathrm{~mm}$ and $4.17 \%$, respectively.

Synthesis of DAA-poly( $N$-alkylacrylamide) (PNAA) CoreShell Type Gel Beads

A typical synthesis of DAA-PNAA core-shell type gel beads was as follows. $30 \mathrm{~mL}$ of a mixed solution of TPP $\left(1.0 \mathrm{~mol} \mathrm{~L}^{-1}\right)$ and NPA $\left(2.0 \mathrm{~mol} \mathrm{~L}^{-1}\right)$ in $N$-methyl-2-pyrrolidone (NMP) was charged into a $50 \mathrm{~mL}$ Erlenmeyer flask in an $80^{\circ} \mathrm{C}$ water bath. Dozens of DAA beads were soaked in this solution for $80 \mathrm{~min}$ until the thickness of the shell layer ( $\left.1-r_{t} / R_{0}\right)$ reached 0.30 , where $R_{0}$ and $r_{t}$ were the external radii of DAA and the unreacted core part when the reaction was carried out for $t \mathrm{~min}$, respectively. The gels were removed from the solution and placed in a large quantity of methanol to terminate the reaction. The resulting G-2, DAA-poly $(N-n-$ propylacrylamide) (PNNPA) core-shell type gel beads were washed with methanol in a Soxhlet extractor. They were dried in vacuo at $60^{\circ} \mathrm{C}$ to a constant weight.

\section{Synthesis of PAA-PNAA Core-Shell Type Gel Beads}

The DAA-PNNPA core-shell type gel beads were neutralized with an acetic acid solution in methanol in a Soxhlet extractor under reflux for $2 \mathrm{~d}$, and then washed with methanol in a Soxhlet extractor. The resulting PAA-PNAA core-shell type gel beads were dried carefully and slowly, and finally dried in vacuo at $60^{\circ} \mathrm{C}$ to constant weight.

\section{Synthesis of PNAA(2)-PNAA(1) Core-Shell Type Gel Beads}

A typical synthesis of PNAA(2)-PNAA(1) core-shell type gel beads was as follows. In NMP, $200 \mathrm{~mL}$ of a mixed solution of TPP $\left(1.0 \mathrm{~mol} \mathrm{~L}^{-1}\right)$ and IPA $\left(2.0 \mathrm{~mol} \mathrm{~L}^{-1}\right)$ was charged into a $300 \mathrm{~mL}$ Erlenmeyer flask in an $80^{\circ} \mathrm{C}$ water bath. Dozens of G-2 samples were soaked in this solution for $24 \mathrm{~h}$. The gels were removed from the solution and placed in a large quantity of methanol to terminate the reaction. The resulting PNAA(2)PNAA(1) core-shell type gel beads were washed with methanol in a Soxhlet extractor. They were dried in vacuo at $60^{\circ} \mathrm{C}$ to a constant weight.

\section{Measurement of Amidation Rate of DAA Bead and DAA- PNAA Core-Shell Type Gel Beads with Alkylamine}

In a typical amidation of dried G-2, a mixed solution $(50 \mathrm{~mL})$ of IPA $\left(2.0 \mathrm{~mol} \mathrm{~L}^{-1}\right)$ and TPP $\left(1.0 \mathrm{~mol} \mathrm{~L}^{-1}\right)$ in NMP was charged into a $50 \mathrm{~mL}$ cylindrical cell in an $80^{\circ} \mathrm{C}$ water bath. A DAA bead was immersed in this solution. The radii of shells $\left(R_{0}\right)$ and cores $\left(r_{t}\right)$ were determined periodically, using a digital video camera (Sony, DCR-TRV950).

\section{Measurement of Swelling/De-Swelling Rate}

A sample was placed in a $50 \mathrm{~mL}$ cylindrical cell at the desired temperature. The change in external radius $\left(R_{t}^{\prime} / R_{D}^{\prime}\right.$ or $\left.R_{t}^{\prime} / R_{0}^{\prime}\right)$, where $2 R_{D}^{\prime}, 2 R_{0}^{\prime}$, and $2 R_{t}^{\prime}$ were the external diameters of the dried gel, when the gel was stored in water at $50{ }^{\circ} \mathrm{C}$, and when the gel swelled or de-swelled for $t$ min, respectively, all were recorded using the digital video camera system described above.

\section{Measurement of Equilibrium Swelling Ratio}

A sample was immersed in water at the desired temperature (the initial temperature was $50^{\circ} \mathrm{C}$ ). The equilibrium-swelling ratio was measured using a previously reported method. ${ }^{9 \mathrm{~b}}$ The relationship $(W s+W p) / W p$ was used, where $W s$ and $W p$ are the weights of the absorbed water and dried polymer, respectively.

\section{RESULTS AND DISCUSSION}

\section{Synthesis of DAA Beads}

DAA beads were prepared via a two-step procedure involving the sedimentation copolymerization of acrylic acid with $0.5 \mathrm{~mol} \%$ of MBAA using the same concentrations of an aqueous AA and MBAA solution as those in the corresponding solution polymerization reported previously, ${ }^{9}$ and neutralization of the resulting gel with excess DBU. The sedimentation polymerization of the aqueous AA solution containing $0.5 \mathrm{~mol} \% \mathrm{MBAA}$ as a crosslinker was carried out using a simple sedimentation polymerization apparatus, consisting of a $500 \mathrm{~mL}$ gas-washing bottle $15 \mathrm{~cm}$ in height and highly viscous silicone oil (kinematic viscosity: 3000 $\mathrm{mm}^{2} \mathrm{~s}^{-1}$ at $25^{\circ} \mathrm{C}$ ) as the heating medium in a water bath at $80{ }^{\circ} \mathrm{C}$, as reported previously. ${ }^{16}$ When the aqueous AA solution was added dropwise to the silicone oil, a droplet (diameter: approximately $3 \mathrm{~mm}$ ) of the monomer solution sedimented slowly into the silicone oil, and it took about $15 \mathrm{~min}$ to reach the bottom of the apparatus. To polymerize perfectly, the droplets stood in the bottom overnight. The resulting PAA beads were neutralized with an excess amount of DBU solution in methanol to give DAA beads. The particle size distribution of the obtained DAA beads is shown in Figure 1. The average diameters $\left(\overline{2 R_{0}}\right)$ and the relative standard deviation $(\delta)$ of the obtained beads were $2.18 \mathrm{~mm}$ and $4.17 \%$, respectively. Millimeter size DAA beads with a very narrow size distribution can thus be obtained by sedimentation polymerization, followed by neutralization with excess DBU.

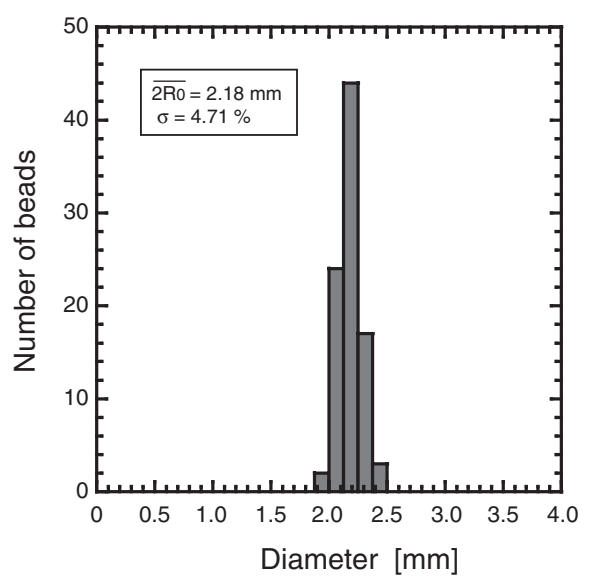

Figure 1. Typical particle size distribution of DAA beads. 

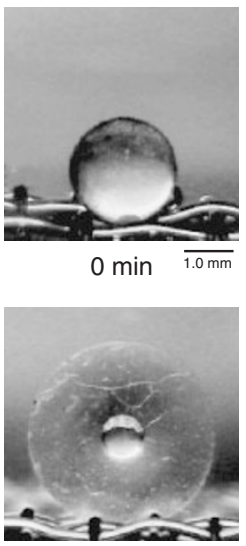

$140 \mathrm{~min}$

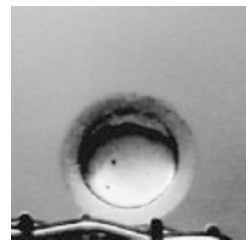

$20 \min$

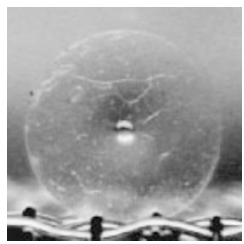

$170 \min$

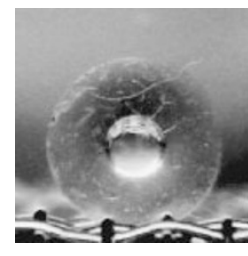

$90 \mathrm{~min}$

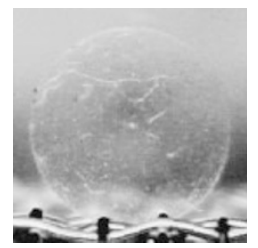

$190 \min$
Figure 2. Typical pictures in the reaction of a DAA bead $\left(2 R_{0}=2.17 \mathrm{~mm}\right)$ with IPA.

\section{Amidation of DAA and DAA-PNAA Core-Shell Type Gel Beads with Alkylamines}

The heterogeneous amidation of a DAA bead with alkylamines $\left(2.0 \mathrm{~mol} \mathrm{~L}^{-1}\right)$ such as IPA and NPA was carried out in NMP using TPP at $80^{\circ} \mathrm{C}$ under the same conditions as that of cylindrical DAA., ${ }^{911}$ The reaction process was visually observed with a video camera. Typical photographs of the amidation are shown in Figure 2. Figure 2 shows that the swelling occurred from the outside, and the bead was divided into a swollen (reacted) outer shell and unswollen (unreacted) inner core because DAA does not swell in NMP and the highly amidated part become swelled in NMP. The inner diameter of the bead decreased and the external diameter increased with increased reaction time. The unreacted core maintained an exactly spherical shape during the reaction, while the core was transformed into an hourglass shape using pellet-type DAA. ${ }^{9 b}$ Thus, it is easy to measure the inner diameter $\left(2 r_{t}\right)$ when the reaction was carried out for $t$ min. Typical rates of disappearance of the core $\left(r_{t} / R_{0}\right)$ are shown in Figure 3. This reaction behavior resembled the corresponding behavior of pellet type DAA with alkylamine, ${ }^{9}$ although the reaction was slightly affected by the shape of DAA. This reaction is markedly affected by the mass transfer rate of reactants and the solvent through the reacted shell layer, and may be sensitive to the internal structure. Since the sedimentation polymerization is very rapid polymerization, some unevenness and strain may result in the DAA beads. There is concern that these factors may disturb the smooth amidation. However, these results suggest that the reaction of the DAA beads proceeded isotropically and smoothly, and the beads had sufficient consistency in this amidation.

The reaction was completed when the unreacted core disappeared. The core disappeared after about $190 \mathrm{~min}$ when a DAA bead $\left(2 R_{0}=2.18 \mathrm{~mm}\right)$ was used. The time $(\tau)$ required for complete disappearance of the unswollen core was about 1160 min. ${ }^{9 \mathrm{~d}}$ when a cylindrical DAA gel (length and diameter: $2 R_{0}=c a$. $5.3 \mathrm{~mm}$ ) prepared by the corresponding normal solution polymerization of AA containing $0.5 \mathrm{~mol} \% \mathrm{MBAA}$

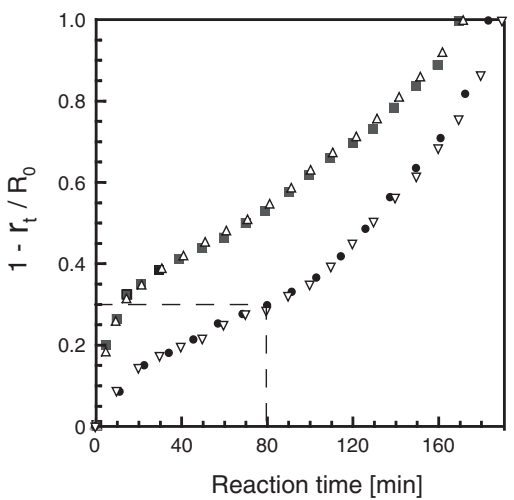

Figure 3. Typical amidation of a DAA bead and DAA-PNAA type gel beads with NPA or IPA; $(\nabla)$ first reaction of DAA with IPA; $(0)$ first reaction of DAA with NPA; $(\triangle)$ second reaction of G-2 with NPA; ( $\mathrm{\square})$ second reaction of G-4 with IPA.

Table I. Synthesis of DAA-PNAA type gel beads by the first amidation of DAA with alkylamine

\begin{tabular}{cllccc}
\hline Run No. & Gel bead & Alkylamine & Solvent & $\begin{array}{c}\text { Reaction time } \\
(\min )\end{array}$ & $r_{t} / R_{0}$ \\
\hline 1 & PNIPA & IPA & NMP & 190 & 0 \\
2 & PNNPA & NPA & NMP & 190 & 0 \\
3 & G-1 & IPA & NMP & 12 & 0.90 \\
4 & G-2 & IPA & NMP & 80 & 0.70 \\
5 & G-3 & NPA & NMP & 12 & 0.90 \\
6 & G-4 & NPA & NMP & 80 & 0.70 \\
\hline
\end{tabular}

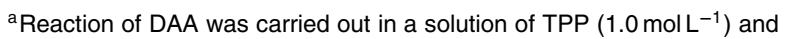
alkylamine $\left(2.0 \mathrm{~mol} \mathrm{~L}^{-1}\right)$ in a solvent $(50 \mathrm{~mL})$ at $80^{\circ} \mathrm{C}$.

was used. As the reaction proceeded under diffusion control of the shrinking-core model, $\tau$ was proportional to $R_{0}^{2}{ }^{10 \mathrm{~b}}$ The $\tau$ $(196 \mathrm{~min})$ of the DAA gel bead $\left(2 R_{0}=2.18 \mathrm{~mm}\right)$ calculated using this equation was a good match for the observed $\tau$ (190 min), although these samples differed in shape. The beads may be equal in quality to the cylindrical gels prepared by the corresponding solution polymerization because the same concentrations of AA and MBAA were used.

The rate was not affected by the type of alkylamine used, because the reaction rate is independent of the concentration. ${ }^{9}$ The complete reaction with IPA and NPA for $190 \mathrm{~min}$ gave PNIPA and PNNPA gel beads, respectively. The thickness of the shell layer can be controlled by the reaction time. DAAPNIPA core-shell type gel beads G-1, G-2 $\left[r_{t} / R_{0}=0.90\right.$ and 0.70] and DAA-PNNPA core-shell type gel beads G-3, G-4 $\left[r_{t} / R_{0}=0.90\right.$ and 0.70$]$ were synthesized by the first amidation of DAA with the corresponding alkylamine using TPP in NMP at $80^{\circ} \mathrm{C}$ for 12 and $80 \mathrm{~min}$., respectively (Table I). Since the unreacted core in the DAA-PNAA core-shell type gel bead is the same as the original DAA sample, it can react with various reagents. When the resulting DAA-PNAA core-shell type gel beads (G-1 and G-3, $r_{t} / R_{0}=0.90$ ) were washed with methanol containing acetic acid in a Soxhlet extractor, neutralization occurred to give the corresponding PAA-PNAA core-shell type gel beads G-5 and G-6 $\left(r_{t} / R_{0}=0.90\right)$, respectively. The second amidation of the dried beads (G-2 and G-4, $r_{t} / R_{0}=$ 0.70 ) with another alkylamine was carried out for $3 \mathrm{~h}$ under the 
Table II. Synthesis of PAA-PNAA and PNAA(2)-PNAA(1) type gel beads by the second reaction of DAA-PNAA type gel beads with acetic acid ${ }^{\mathrm{a}}$ and alkylamines ${ }^{\mathrm{b}}$

\begin{tabular}{rlll}
\hline $\begin{array}{c}\text { Run } \\
\text { No. }\end{array}$ & \multicolumn{1}{c}{ Gel bead } & Reagent & $\begin{array}{c}\text { DAA-PNAA type gel bead } \\
\left(r_{\mathrm{t}} / R_{0}\right)\end{array}$ \\
\hline 9 & G-5 (PAA-PNIPA) & $\mathrm{H}^{+\mathrm{a}}$ & $\mathrm{G}-1(0.90)$ \\
10 & G-6 (PAA-PNNPA) & $\mathrm{H}^{+\mathrm{a}}$ & $\mathrm{G}-3(0.90)$ \\
11 & G-7 (PNNPA-PNIPA) & NPA $^{\mathrm{b}}$ & $\mathrm{G}-2(0.70)$ \\
12 & G-8 (PNIPA-PNNPA) & IPA $^{\mathrm{b}}$ & $\mathrm{G}-4(0.70)$ \\
\hline
\end{tabular}

a Neutralization of DAA-PNAA type gel bead with an acetic acid solution in methanol was carried out in a Soxhlet extractor under reflux for $2 \mathrm{~d}$. ${ }^{b}$ Amidation of DAA-PNAA type gel bead was carried out in a solution of TPP $\left(1.0 \mathrm{~mol} \mathrm{~L}^{-1}\right)$ and alkylamine $\left(2.0 \mathrm{~mol} \mathrm{~L}^{-1}\right)$ in a solvent at $80^{\circ} \mathrm{C}$ for $24 \mathrm{~h}$.

same conditions as the first amidation. After rapid swelling of the amidated shell layer at the beginning, the second amidation proceeded (Figure 3). The core disappeared after about $170 \mathrm{~min}$. The $\tau$ was only slightly shorter than that of the first amidation. The same results were observed in the corresponding reaction of cylindrical DAA. ${ }^{11}$ The complete amidation of G-2 and G-4 $\left(r_{t} / R_{0}=0.70\right)$ gave the corresponding PNAA(2)PNAA(1) core-shell type gel beads, G-7 and G-8, respectively (Table II).

\section{Characterization of the Gel Beads Obtained by the Second Amidation}

The typical FT-IR spectra of core and shell in a G-7 bead and a PAA gel bead are shown in Figure 4. The amidated parts showed strong absorption peaks at $3300 \mathrm{~cm}^{-1}(\mathrm{~N}-\mathrm{H}$, stretching), 1650 and $1545 \mathrm{~cm}^{-1}$ (amide $\mathrm{I}$ and amide II) with no absorption occurring at $1755 \mathrm{~cm}^{-1}(\mathrm{C}=\mathrm{O}$ of phenyl ester). The shell layer and core of the core-shell type gel beads showed absorption peaks characteristic of PNIPA at 1385, 1368, 1173 and $1131 \mathrm{~cm}^{-1}$ and of PNNPA at 1384 and $1153 \mathrm{~cm}^{-1}$; the absorption spectra were clearly consistent with those of PNIPA and PNNPA gels prepared by radical polymerization, ${ }^{8}$ as well as the corresponding cylindrical gels, ${ }^{11}$ respectively. The spectra appear to have trace absorption at $1710 \mathrm{~cm}^{-1}(\mathrm{C}=\mathrm{O}$ of carboxylic acid). The ratio of absorption at $1710 \mathrm{~cm}^{-1}$ to that at $1650 \mathrm{~cm}^{-1}$ was smaller than that of soluble PNIPA film containing $1 \mathrm{~mol} \%$ PAA. These results indicate that the second amidation of DAA-PNAA core-shell type gel beads with an alkylamine was nearly quantitative, giving the corresponding PNAA(2)-PNAA(1) core-shell type gel beads.

\section{Thermal Swelling/De-Swelling Behavior of the Homo- polymer Gel Beads}

PNAA gels prepared by the amidation of DAA have great merit; these gels showed higher thermosensitivity than those prepared by the polymerization of the corresponding monomer. ${ }^{11}$ The equilibrium-swelling ratio for PNIPA and PNNPA gel beads was measured in water over a wide temperature range (Figure 5). The PNIPA and beads PNNPA showed the maximal equilibrium-swelling ratio at a temperature several degrees lower than their lower critical solution temperatures (LCSTs), where these beads hardly swelled at a temperature above the LCST. In addition, the beads had LCSTs of approximately

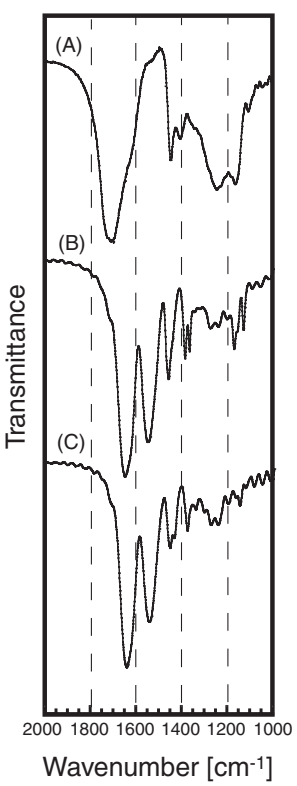

Figure 4. IR spectra $(\mathrm{KBr})$ of (A) PAA bead; (B) PNIPA shell of G-7, and (C) PNNPA core of G-7.

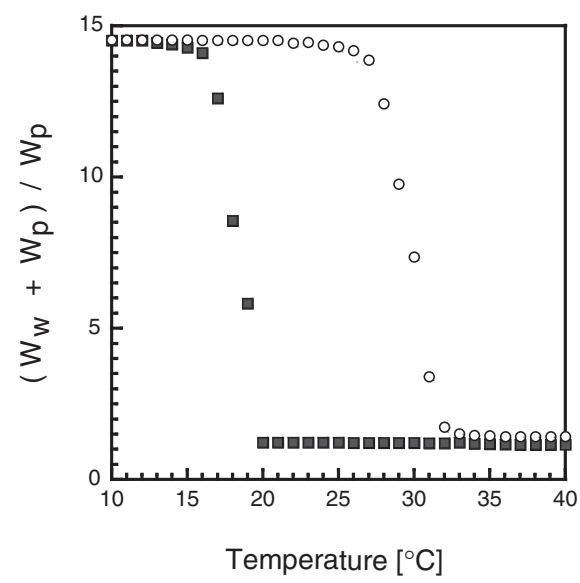

Figure 5. Equilibrium swelling ratio of PNAA gels in water as a function of temperature; ( $\square$ ) PNNPA; (○) PNIPA.

$32{ }^{\circ} \mathrm{C}$ and $20^{\circ} \mathrm{C}$, which are the same as those prepared by polymerization $^{17}$ as well as the corresponding cylindrical gels, although the LCST of PNAA was very sensitive to small amount of impurities. ${ }^{9 a, 18}$ These results suggested that highly purified and uniform PNAA was obtained by this method.

\section{Thermal Swelling/De-Swelling Behavior of the Core-Shell Type Gel Beads}

The swelling/de-swelling behavior at various temperatures of these new core-shell type gel beads with thermosensitive shell layers is of great interest. At a temperature below the LCSTs of the shell layers, the beads began to swell from the outside, while maintaining an exactly spherical shape during swelling. Therefore, the swelling behavior using the expression $R_{t}^{\prime} / R_{D}^{\prime}$, where $2 R_{D}^{\prime}$ and $2 R_{t}^{\prime}$ are the external diameters of the dried gel and the swollen gel when the swelling was carried out for $t$ min, respectively, could be precisely observed because the 


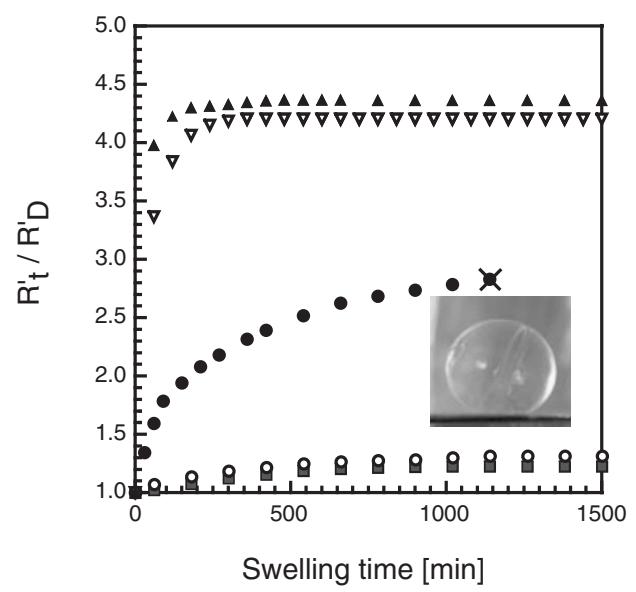

Figure 6. Swelling of beads in water at $50^{\circ} \mathrm{C} ;(\mathbf{A})$ DAA, $(\nabla) \mathrm{G}-1 ;(\mathbf{O})$ G-3, $(\bigcirc)$ G-5; $(\square)$ G-6. The $\times$ mark denotes the occurrence of burst.

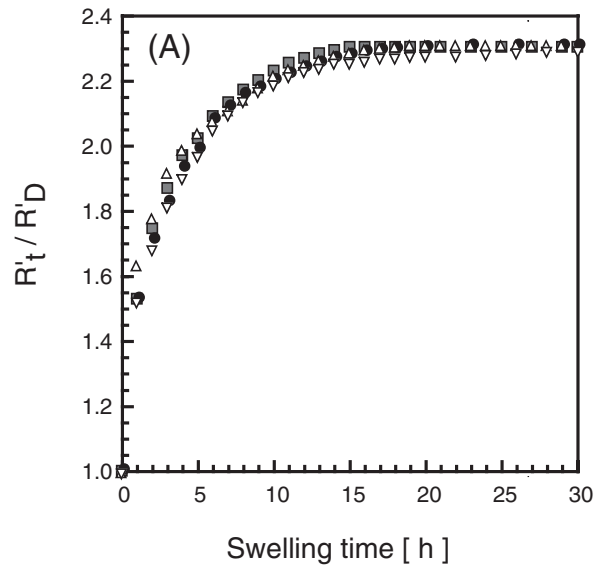

Figure 8. Swelling of various beads;

PNIPA; ( $\boldsymbol{\nabla})$ PNNPA

shape was not distorted. Swelling of the dried beads was carried out in water at $50^{\circ} \mathrm{C}$ above their LCSTs. The gel beads were unswollen and could function as a permeability barrier to water, since the shell layer became hydrophobic (Figure 6). However, the DAA-PNIPA core-shell type gel bead, G-1, underwent rapid swelling in water, and the shell layers could not be differentiated from the very swollen DAA core. The DAA-PNNPA core-shell type gel bead, G-3, swelled gradually and the PNNPA shell layer finally split in two within $24 \mathrm{~h}$, although the spherical shape was not distorted. Similar results were observed in the swelling of the corresponding cylindrical gels. ${ }^{9 \mathrm{e}}$ The DAA core is therefore too hydrophilic to disturb the transition of the thermosensitive PNAA shell layer from hydrophilicity to hydrophobicity.

In contrast to DAA-PNAA core-shell type gel beads, PAAPNAA core-shell type gel beads G-5 and G-6, prepared by the neutralization of the corresponding DAA-PNAA core-shell type gel beads, hardly swelled because the shell layers cannot swell in water. Typical swelling behavior of G-6 at various temperatures is shown in Figure 7. At $15^{\circ} \mathrm{C}$ below the LCST, G-6 swelled in water and the equilibrium swelling ratio was equal to PAA $\left(R_{t}^{\prime} / R_{D}^{\prime}=c a .2 .7\right.$ at $\left.15^{\circ} \mathrm{C}\right)$. At $25^{\circ} \mathrm{C}$ and $30^{\circ} \mathrm{C}$

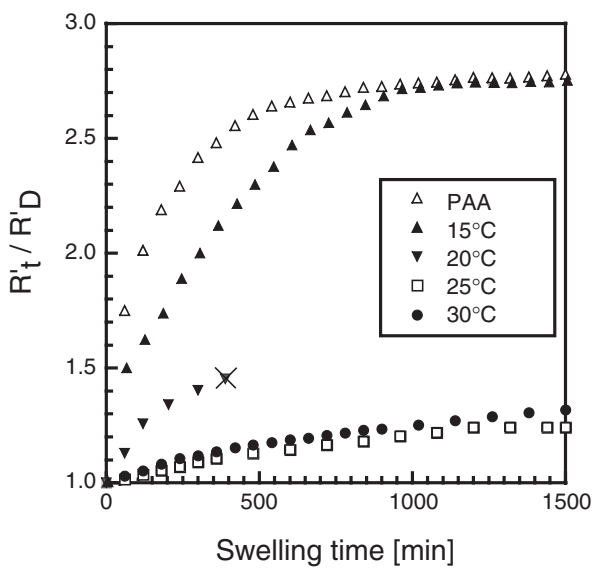

Figure 7. Swelling of PAA bead and G-6 bead in water; $(\triangle)$ PAA at $15^{\circ} \mathrm{C}$; (A) G-6 at $15^{\circ} \mathrm{C} ;(\boldsymbol{\nabla})$ G-6 at $20^{\circ} \mathrm{C} ;(0)$ G-6 at $25^{\circ} \mathrm{C}$; ( $\left.\square\right)$ G-6 at $30^{\circ} \mathrm{C}$. The $\times$ mark denotes the occurrence of burst.

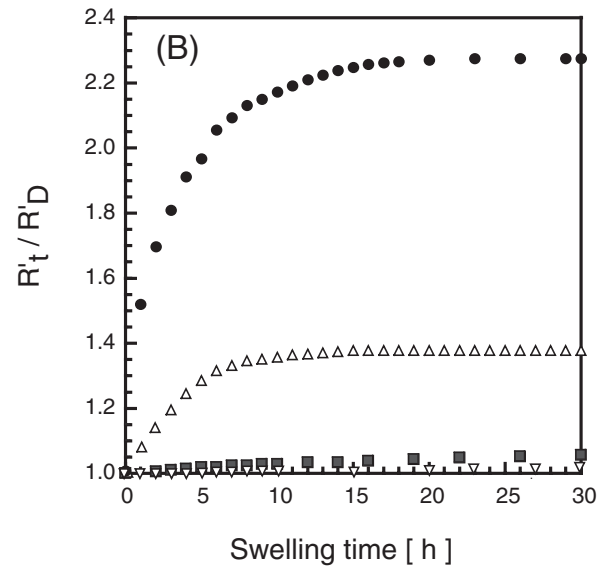

; $\triangle$ ) G-7; $(\square)$ G-8 (A) in water at $15^{\circ} \mathrm{C}$; (B) in water at $25^{\circ} \mathrm{C}$.

above the LCST, G-6 swelled only slightly, and the de-swollen PNNPA shell layer depressed the swelling of the core, although the shell layer was broken at $20^{\circ} \mathrm{C}$ just on the LCST. The same results were observed in the swelling of the corresponding cylindrical core-shell type gels. ${ }^{9 b, d}$ These results suggest that the PNAA shell layer in the PAA-PNAA core-shell type bead can function as a permeability barrier to water at a temperature above the LCST, whereas the PNAA shell layer in the DAAPNAA core-shell type bead did not work. In each case, the bead maintained its exact spherical shape, while the cylindrical dried gel had some dimples on its surface and the cylindrical shape was distorted during the swelling. ${ }^{9 b}$

The swelling/de-swelling behavior of PNAA(2)-PNAA(1) core-shell type gel beads, as well as PNIPA and PNNPA gel beads, was measured in water at three temperatures $(15,25$, and $35^{\circ} \mathrm{C}$ ). Swelling of the dried PNAA(2)-PNAA(1) coreshell type gel beads was carried out in water at $15^{\circ} \mathrm{C}$ below their LCSTs. All the gel beads showed the same swelling behavior, that is, these beads swelled slowly but maintained their spherical shape, and reached the equilibrium swelling ratio after $20 \mathrm{~h}$ (Figure 8), while they did not swell at a temperature above $32^{\circ} \mathrm{C}$, LCST of PNIPA. At the middle 


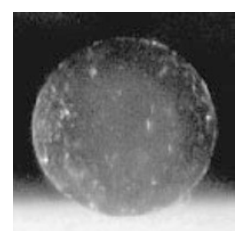

G-7 swollen at $15^{\circ} \mathrm{C}$ $0 \mathrm{~min}$

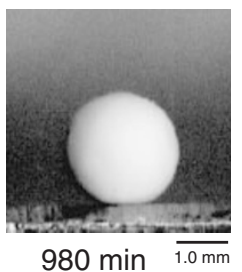

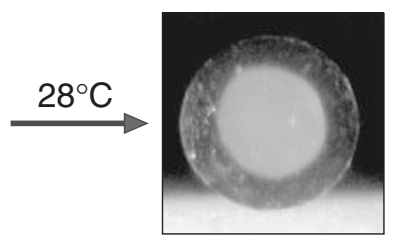

$20 \min$

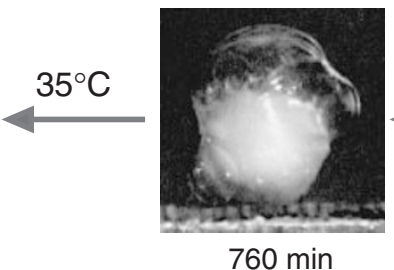

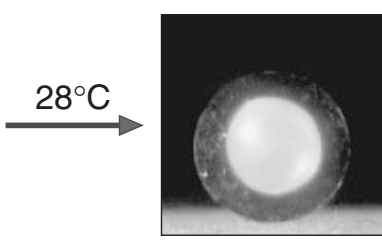

$600 \mathrm{~min}$

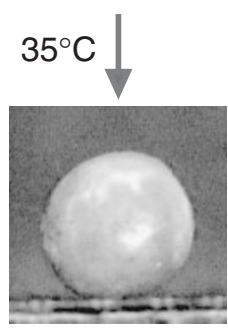

$601 \mathrm{~min}$

Figure 9. Typical pictures in the de-swelling of a G-7 bead previously swelled in water, in response to stepwise changes in temperature.

temperature between the LCSTs of PNIPA and PNNPA, marked differences in swelling behaviors among these beads were observed in water. The PNNPA gel bead and PNNPA shell layer in G-7 swelled well at $25^{\circ} \mathrm{C}$. The PNIPA beads and PNIPA core in G-7 did not swell. G-8 hardly swelled, because the PNIPA shell did not swell, and depressed the swelling of the PNNPA core.

The de-swelling of G-7, a PNNPA-PNIPA core-shell type gel $\left[r_{t} / R_{0}=0.90\right]$, swollen in water at $15^{\circ} \mathrm{C}$, was carried out at different temperatures. Typical images of de-swelling are shown in Figure 9. Shortly after the gel was immersed in water at $28^{\circ} \mathrm{C}$, the light milky PNNPA core appeared in the gel. The PNNPA core shrunk gradually while the PNIPA shell kept swelling. The external diameter $\left(R_{t}^{\prime} / R_{0}^{\prime}\right)$ of the gel, as well as the core, where $R_{0}^{\prime}$ and $R_{t}^{\prime}$ are the external diameters of the gel stored in water at $50^{\circ} \mathrm{C}$ and de-swelled in water for $t$ min, respectively, decreased slowly, but the beads maintained their spherical shape, and reached the equilibrium swelling ratio after $180 \mathrm{~min}$ (Figure 10). A previous paper ${ }^{9 \mathrm{~d}}$ reported that the swelled PNIPA and PNNPA gels were produced in a dense skin layer when the swollen beads were dipped into the water at a temperature above their LCSTs; however, these gels differed in their de-swelling behavior. The de-swelling rate for the PNNPA gel decreased with increasing water temperature because the skin layer became tougher, while the rate for the PNIPA gel increased with increasing water temperature because the shrinkage force became stronger. However, the de-swelling of the PNNPA core at $28^{\circ} \mathrm{C}$ is much higher than that at $25^{\circ} \mathrm{C}$. It seems that the swollen PNIPA shell may prevent the formation of a dense skin layer on the PNNPA core. When the gel that stood for a long time at $28^{\circ} \mathrm{C}$ was dipped in water at $35^{\circ} \mathrm{C}$, a dense skin layer, which functions as a water permeability barrier, was immediately produced on the shell layer. The shrinkage force was so strong that water was ejected from the inside of the gel to produce some bubbles on the surface-the squeeze effect, ${ }^{9 e, 18}$ and the gel finally shrank (Figure 10). G-8, a PNIPA-PNNPA core-shell type gel bead,

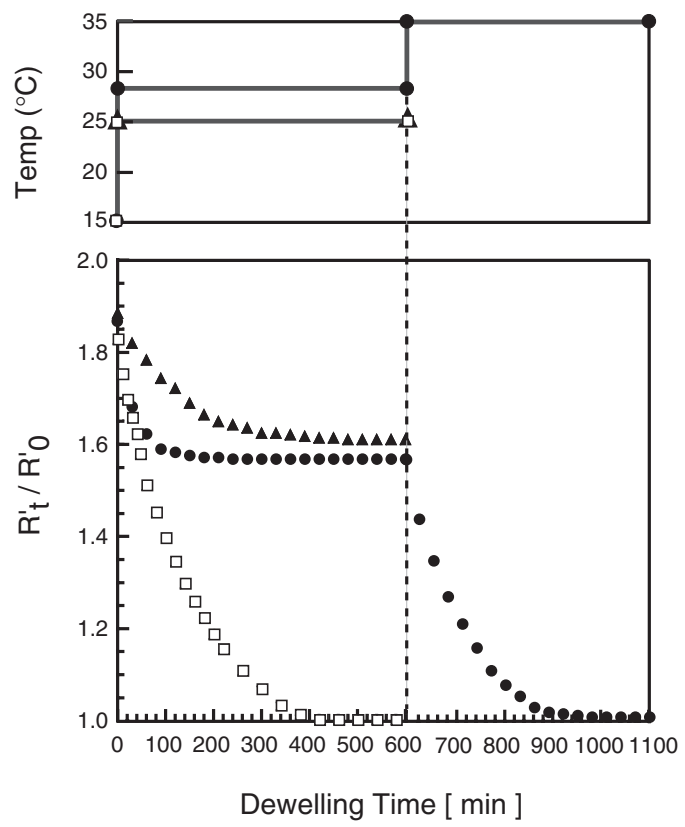

Figure 10. De-swelling of gel beads previously swelled in water in response to stepwise changes in temperature; ( $\square$ ) PNNPA at $15 \rightarrow 25^{\circ} \mathrm{C}$ (A) G-7 at $15 \rightarrow 25^{\circ} \mathrm{C}$; (O) G-7 at $15 \rightarrow 28 \rightarrow 35^{\circ} \mathrm{C}$.

showed swelling/de-swelling behavior similar to that of G-7, and the external diameter decreased in response to the stepwise temperature changes because the shell layer and core in the bead could occur independently. ${ }^{11}$ However, the PNNPA shell layer became clouded and the swelling/de-swelling of the PNIPA core was not directly observed when G-8 was placed in water at temperatures above the LCST of PNNPA.

\section{CONCLUSION}

DAA gel beads were prepared via a two-step procedure involving the sedimentation copolymerization of acrylic acid 
with $0.5 \mathrm{~mol} \%$ of MBAA and neutralization of the resulting gel with excess DBU in methanol. The first amidation of DAA beads with alkylamines proceeded at $80^{\circ} \mathrm{C}$ in the presence of TPP to give the corresponding DAA-PNAA core-shell type gel beads. The thickness of the shell layer could be controlled by the reaction time. PAA-PNAA core-shell type gel beads and PNAA(2)-PNAA(1) core-shell type gel beads were prepared by further reaction of DAA-PNAA core-shell type gel beads with acid and another alkylamine, respectively. The independent thermosensitive behavior of each block in double thermosensitive block copolymers has been reported. ${ }^{12-14}$ Since the PNAA(2)-PNAA(1) and PAA-PNAA core-shell type gel beads had a defined structure - a continuous network and a boundary between two layers, corresponding to well-controlled crosslinking block copolymers-the swelling/de-swelling of the thermosensitive shell layer and core in the gel beads also occurred independently in response to the temperature changes.

The amidation of the DAA beads and the swelling/deswelling of the amidated gel beads occurred isotropically and the beads maintained their spherical shapes, except for a case of appearance of the squeeze effect, where the cylindrical gels were distorted. Therefore, these behaviors can be precisely measured using spherical gels. However, a large difference in those behaviors between cylindrical gels and gel beads was not observed. These results indicate that the beads prepared by sedimentation polymerization may be equal in quality to the cylindrical gels prepared by the corresponding solution polymerization, and have enough uniformity to be available starting materials for chemical modification.

Acknowledgment. This work was supported by a grant from the Ministry of Education, Science and Culture of Japan (No. 18550195 and No. 20550189), which is gratefully acknowledged.
Received: April 22, 2009

Accepted: July 4, 2009

Published: August 19, 2009

\section{REFERENCES}

1. E. Ruckenstein and I. Hong, Polymer, 36, 2857 (1995).

2. H. Zhang and A. I. Cooper, Chem. Mater., 14, 4017 (2002).

3. H. Zhang and A. I. Cooper, Macromolecules, 36, 5061 (2003).

4. T. Iizawa, T. Ishido, T. Gotoh, and S. Sakohara, Polym. J., 39, 18 (2007).

5. E. Ruckenstein and I. Hong, J. Appl. Polym. Sci., 61, 1949 (1996).

6. E. Ruckenstein and I. Hong, Chem. Mater., 8, 546 (1996).

7. T. Iizawa, T. Ninomiya, T. Gotoh, and S. Sakohara, Polym. J., 36, 356 (2004).

8. T. Iizawa, T. Ishido, T. Gotoh, and S. Sakohara, J. Appl. Polym. Sci., 104, 842 (2007).

9. a) T. Iizawa, N. Matsuno, M. Takeuchi, and F. Matsuda, Polym. J., 31, 1277 (1999).

b) T. Iizawa, N. Matsuno, M. Takeuchi, and F. Matsuda, Polym. J., 34, 63 (2002).

c) T. Iizawa, Y. Matsuura, K. Hashida, and Y. Onohara, Polym. J., 35, 815 (2003).

d) T. Iizawa, Y. Matsuura, and Y. Onohara, Polymer, 46, 8098 (2005).

10. a) S. Yagi and T. Kunii, Kogyo Kagaku Zasshi, 56, 131 (1953).

b) "Chemical Reaction Engineering," 3rd ed, O. Levenspiel Ed., John Wiley \& Sons, New York, 1999, p 566.

11. T. Iizawa, A. Terao, M. Ohuchida, Y. Matsuura, and Y. Onohara, Polym. J., 39, 1177 (2007).

12. a) M. Arotçaréna, B. Heise, S. Ishaya, and A. Laschewsky, J. Am. Chem. Soc., 124, 3787 (2002).

b) J. Virtanen, M. Arotçaréna, B. Heise, S. Ishaya, A. Laschewsky, and H. Tenhu, Langmuir, 18, 5360 (2002).

13. J. V. M. Weaver, S. P. Armes, and V. Bütün, Chem. Commun., 2122 (2002).

14. Y. Maeda, H. Mochiduki, and I. Ikeda, Macromol. Rapid Commun., 25, 1330 (2004).

15. T. Iizawa and A. Terao, MRS Proc., 1129, V04-06 (2008).

16. T. Iizawa, K. Nakao, T. Yamaguchi, and M. Maruta, Polymer, 46, 1834 (2005).

17. S. Ito, Kobunshi Ronbunshu, 46, 437 (1989).

18. T. Okano, Y. H. Bae, H. Jacobs, and S. W. Kim, J. Controlled Release, 11, 255 (1990). 